\title{
Blind surveys for radio pulsars and transients
}

\author{
D.R. Lorimer \\ Physics Department, West Virginia University, Morgantown, WV 26506, USA
}

\begin{abstract}
The main reasons for searching for pulsars are to: (i) get an accurate census of the neutron star population and its origin and evolution; (ii) connect neutron stars to other stellar populations in the Galaxy and globular clusters; (iii) study Galactic astronomy (the interstellar medium and magnetic field); (iv) find and study new interesting individual objects; (v) study pulsar phenomenology; (vi) find pulsars to add to the sensitivity of pulsar timing arrays. This review focuses on blind (i.e. large area) searches for radio pulsars. I'll summarize the methods we use, some of the challenges they present, look at some of the recent and current efforts going on. I will also look at outreach of this area to groups outside the traditional area of pulsar research, highlight the discoveries of radio transients and look ahead to the future. Pulsars found at other wavelengths will be reviewed elsewhere in this volume.
\end{abstract}

\section{SEARCH METHODS}

The goal of pulsar searches is to find a dispersed set of pulses buried in a twodimensional data set of radio frequency versus time. This is traditionally achieved by dividing the radio band up into discrete frequency channels which are subsequently dedispersed by appropriately delaying the frequency channels before summing over the whole bandwidth. Since in any given search we do not know the dispersion measure $\mathrm{DM}^{1}$ a-priori, we must create time series corresponding for a range of DMs. To dig out periodic sources from the noise, we Fourier transform each time series to reveal their signature as a series of harmonically related components in the amplitude or power spectrum. We then search these spectra for statistically significant features and repeat this process for all trial DMs to obtain a list of pulsar candidates which are deemed to be statistically significant. For each of these candidates, we return to the original data set and fold it at the nominal DM and period and examine the signal in time and radio frequency. An example set of diagnostic plots showing a pulsar discovery (in this case, from Scott Ransom's PRESTO software package ${ }^{2}$ ) can be seen in Fig. 1.

The other method looks for individual pulses which would not appear as significant features in the Fourier transform search. In this case, one records individual events in each time series which significantly exceed the overall $\mathrm{rms}$ noise level (e.g. $5 \sigma$ or above). The results in this case is a set of diagnostic plots (in this case, from the SIGPROC software package ${ }^{3}$ ) shown in Fig. 2. From these, it is fairly straightforward to

\footnotetext{
${ }^{1}$ Defined, in the usual way, as the integrated column density of free electrons along the line of sight to the pulsar, i.e. $D M=\int_{0}^{d} n_{e} d l$.

$2 \mathrm{http} / / /$ github.com/scottransom/presto

${ }^{3} \mathrm{http}: / /$ sigproc.sourceforge.net
} 


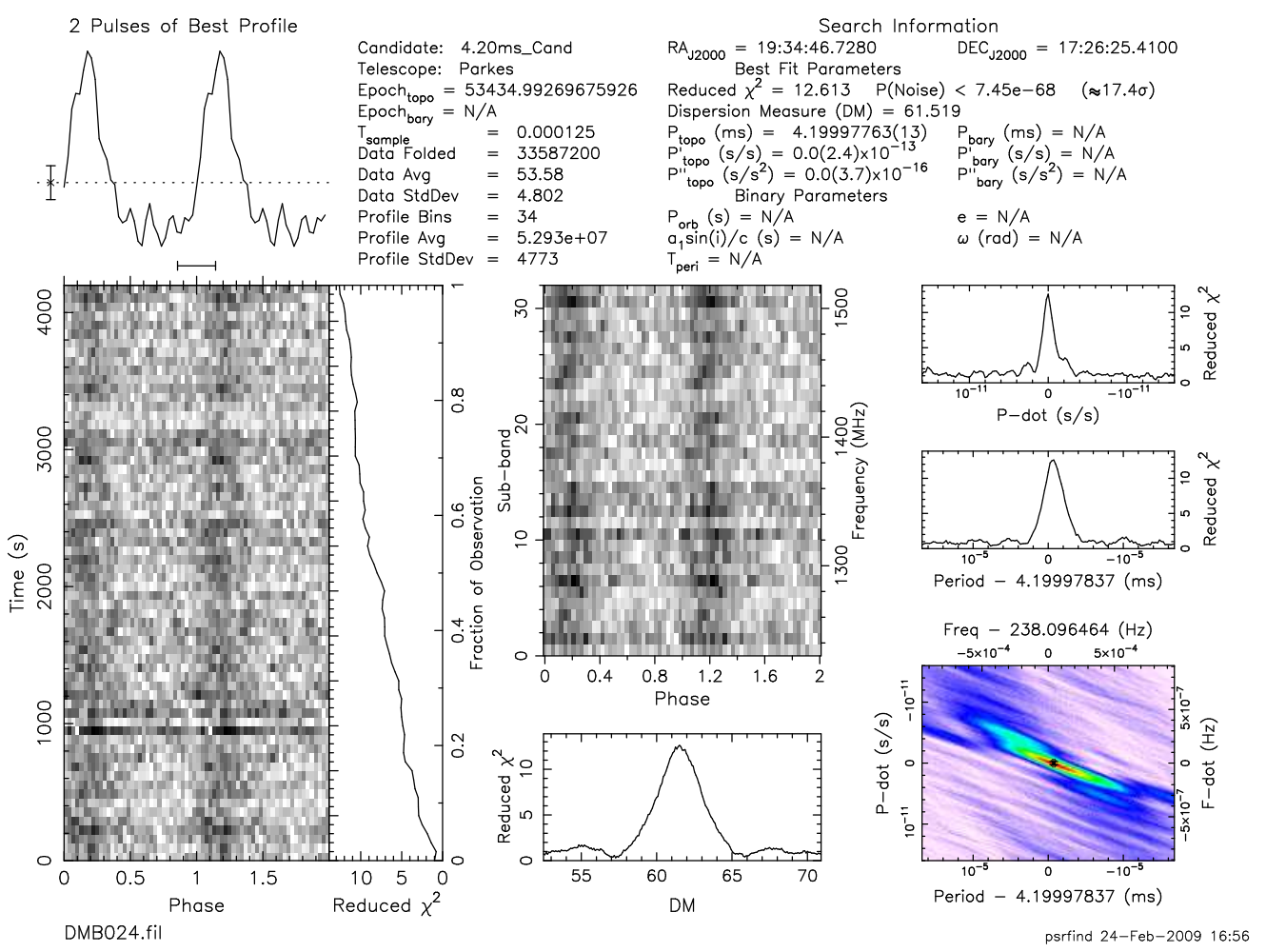

FIGURE 1. Example periodicity search output plot showing data folded in time (lower left) and radio frequency (upper center) as well as the integrated pulse profile (upper left) and optimal DM search (lower center). The statistical significance of the signal in each of these diagrams is measured in terms of the reduced $\chi^{2}$ square value computed from the integrated pulse profiles. A $\chi^{2}$ value close to unity would be found for a profile that is consistent with Gaussian random noise. Shown here is the discovery observation for the 4.2-ms pulsar J1935+1726 from a multibeam survey at Parkes (Camilo et al. 2011).

see whether any significant signals are present in the data. Further details on both search techniques can be found in Lorimer \& Kramer (2005).

\section{FACTORS AFFECTING SEARCH SENSITIVITY}

The discussion here is necessarily brief, and highlights the key limitations on a pulsar search. Further information can be found in Lorimer \& Kramer (2005).

Scintillation produces a diffraction pattern on the observer's plane can result in significant flux density modulation from one epoch to the next. This effect is particularly significant when the scintillation bandwidth is greater than the observing bandwidth. It is particularly prevailant in low-frequency $(\lesssim 1 \mathrm{GHz})$ observations of low-DM $\left(\lesssim 50 \mathrm{~cm}^{-3} \mathrm{pc}\right)$ pulsars and cannot be removed by instrumental means. Multiple observations of the same patch of sky are really the only way to minimize this effect without changing the observing frequency and/or bandwidth of the system. Multiple passes will also help mitigate against pulsar intermittency. 

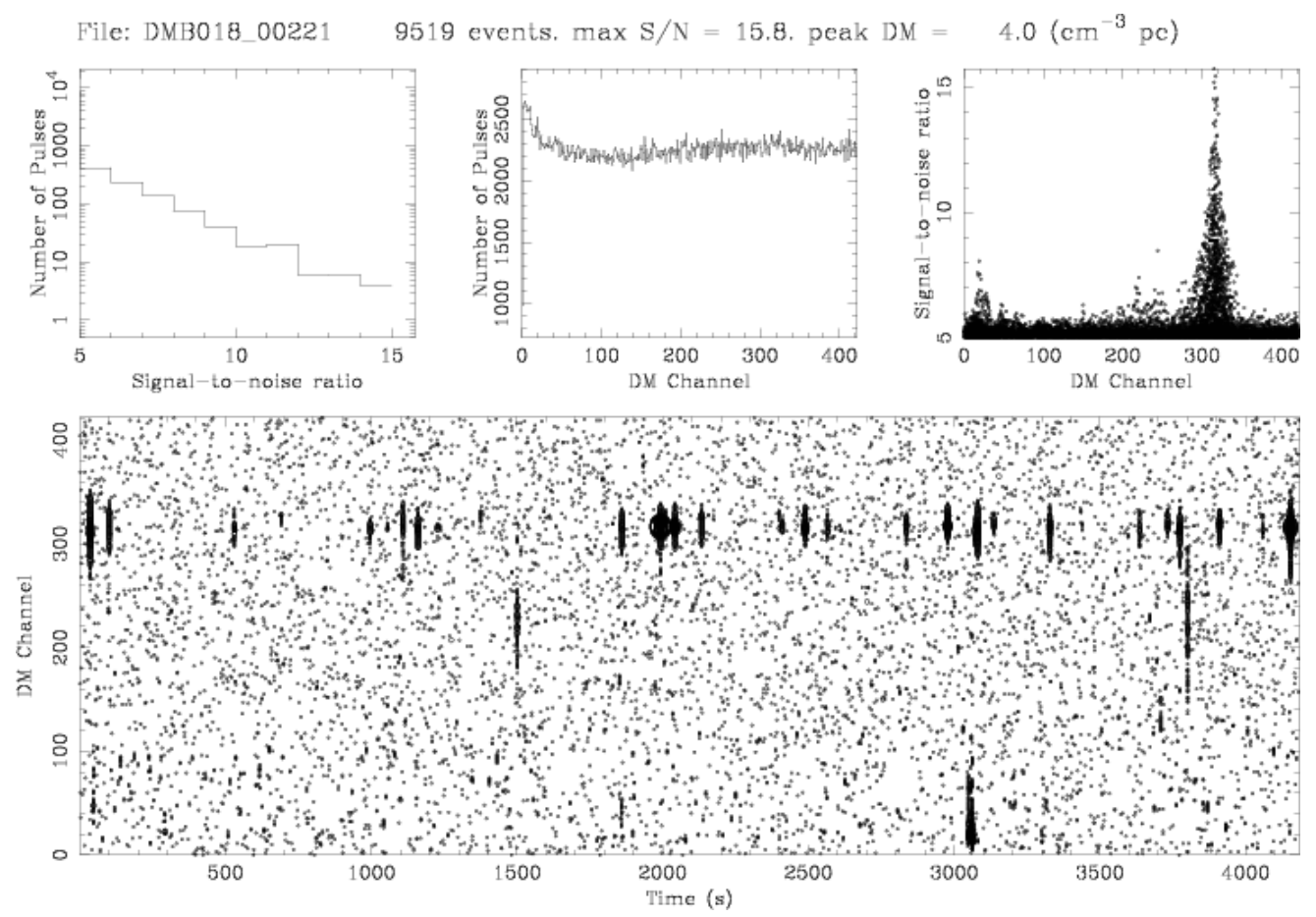

FIGURE 2. Example single pulse search plot, in this case from Maura McLaughlin and Jim Cordes' matched filtering algorithm, showing signal-to-noise of events as a function of trial DM and time (lower plot), distribution of signal-to-noise ratios (upper left), number versus trial DM (center) and signal-tonoise versus trial DM (upper left). Shown here is the discovery observation of the 1.9-s pulsar J1925+19 from a multibeam survey at Parkes (Camilo et al. 2011).

Scattering produced by multi-path propagation as the pulses propagate through a turbulent interstellar medium which results in rays arriving over a variety of path lengths and a classical one-sided exponential to pulse profiles. In extreme cases, the signal-tonoise ratio $(\mathrm{S} / \mathrm{N})$ can be greatly reduced as the broadening timescale approaches a pulse period. While this effect cannot easily be removed via instrumental means, it can be minimized by observing at higher frequencies since the exponential decay time scales approximately as $f^{-4}$, where $f$ is the observing frequency. The trade-off here is that the pulsar flux density spectra are generally very steep, scaling as $f^{-1.5}$.

Sky background produced by free electrons radiating synchrotron emission due to their motion in the Galactic magnetic field is strongly position dependent. In particular, it can add significantly to the overall system noise for observations at low Galactic latitudes and towards the Galactic center. Fortunately the spectrum of this radiation is also quite steep $\left(\sim f^{-1.6}\right)$ so that high-frequency $(f>1 \mathrm{GHz})$ observations generally have only minimal contamination due to this effect.

Binary motion during an integration is becoming increasingly important to account for as we seek the most extreme binaries lurking in the Galaxy. The shift in pulse phase during an observation of even just a few minutes can be very significant even for an orbital period of a few hours. For the most exotic binaries, which might exist with orbital periods of a few 10s of minutes, only by accounting for orbital motion can one hope to 
TABLE 1. Blind pulsar surveys carried out using the $20 \mathrm{~cm}$ Parkes multibeam receiver and now the legendary one-bit filterbank spectrometers. The three pulsars listed in the Magellanic clouds are new discoveries in archival data from a recent analysis by the author and summer student Z. Bailey.

\begin{tabular}{llllr}
\hline \multicolumn{1}{c}{ Surveyed region } & Dwell time & Area $\left(\mathbf{d e g}^{2}\right)$ & Discoveries & Primary reference \\
\hline Galactic plane & $35 \mathrm{~min}$ & 1700 & $>700$ & Manchester et al. (2001) \\
High latitudes & $265 \mathrm{~s}$ & 3600 & 18 & Burgay et al. (2006) \\
Intermediate latitudes & $265 \mathrm{~s}$ & 2000 & 57 & Edwards et al. (2001) \\
High latitudes & $265 \mathrm{~s}$ & 4150 & 26 & Jacoby et al. (2009) \\
Northern Galactic plane & $70 \mathrm{~min}$ & 40 & 18 & Camilo et al. (2011) \\
Perseus spiral arm & $35 \mathrm{~min}$ & 500 & 13 & Burgay et al. (2011) \\
Magellanic clouds & $2.3 \mathrm{hr}$ & 270 & $14+3$ & Manchester et al. (2006) \\
\hline
\end{tabular}

detect such a pulsar. These "acceleration searches" are carried out in either the time or frequency domain and attempt to remove the effects of orbital motion as completely as possible. In the simplest case, the pulsar's motion is approximated as a constant acceleration during the orbit and this can result in substantial gains in search sensitivity. For shorter orbital periods, where this approximation breaks down, more sophisticated methods are required. One such example is the demodulation approach being carried out by the EINSTEIN@Home team on the PALFA data (Knipsel et al. 2010; see also the contribution by Lazarus in these proceedings). This approach shows great promise and should result in some very exciting discoveries over the next few years.

\section{PAST AND PRESENT SURVEYS}

Many of the blind survey discoveries during the period 1998-2008 were made by a variety of projects which took advantage of the Parkes $20 \mathrm{~cm}$ multibeam receiver (Staveley-Smith et al. 1996). The 13 cooled receivers on the sky allowed simultaneously a quantum leap in survey speed and sensitivity over most previous pulsar surveys. It has so far resulted in the discovery of over 1000 pulsars. The various surveys responsible for this revolution in discovery space are summarized in Table 1.

Motivated by these discoveries, we are now enjoying an era of high-sensitivity surveys carried out at a variety of telescopes. These surveys are summarized in Table 2. The results from Parkes, where the High Time Resolution Universe (HTRU) survey are now being undertaken using a new generation of spectrometers on the Parkes multibeam system are discussed by Keith and Bates in these proceedings. Further contributions discussing the Green Bank drift-scan survey (Boyles) and the Pulsar Arecibo L-Band Feed Array (PALFA) survey (Lazarus) are elsewhere in this volume. The Giant Metre Wave Radio telescope (GMRT) is conducting a number of surveys at frequencies in the range 0.3-1.4 GHz. At Effelsberg, a northern complement of the HTRU survey is being carried out (Barr, these proceedings). The Green Bank Northern Celestial Cap (GBNCC) survey is an ambitious project to survey the entire sky north 38 degrees in declination at $350 \mathrm{MHz}$ using the GBT. A large number of new discoveries are expected from these surveys over the next five years. 
TABLE 2. The current generation of blind pulsar surveys carried out at a variety of observatories and frequencies. Those surveys with zero discoveries listed have only recently begun data collection. Those surveys with $>$ signs listed are ongoing and further discoveries are expected soon.

\begin{tabular}{llllr}
\hline \multicolumn{1}{c}{ Survey name } & Telescope & Frequency & Discoveries & Primary reference \\
\hline High Resolution Universe & Parkes & $1.4 \mathrm{GHz}$ & $>65$ & Keith et al. (2010) \\
Eight-grate Cygnus survey & Westerbork & $328 \mathrm{MHz}$ & $>3$ & Janssen et al. (2009) \\
Methanol Multibeam & Parkes & $6.8 \mathrm{GHz}$ & 3 & Bates et al. (2010) \\
Northern plane & GBT & $350 \mathrm{MHz}$ & $>33$ & Hessels et al. (2009) \\
Drift-Scan & GBT & $350 \mathrm{MHz}$ & $>27$ & Boyles (these proceedings) \\
Arecibo L-Band Feed Array & Arecibo & $1.4 \mathrm{GHz}$ & $>55$ & Cordes et al. (2006) \\
High Resolution Universe & Effelsberg & $1.4 \mathrm{GHz}$ & 0 & Barr (these proceedings) \\
Northern Celestial Cap & GBT & $350 \mathrm{MHz}$ & 0 & Ransom et al. (2015) \\
Galactic plane & GMRT & $610 \mathrm{MHz}$ & $>3$ & Joshi et al. (2009) \\
\hline
\end{tabular}

\section{OUTREACH EFFORTS}

A number of groups have been recently been engaging in involving high-school and undergraduate students in pulsar research. In the Pulse@ Parkes project (Hobbs et al. 2010), high-school students carry out regular timing observations of pulsars and learn about their properties. The Arecibo Remote Control Center project ${ }^{4}$ (ARCC; Miller et al. 2009) allows high-school and college students to search for pulsars and observe with the Arecibo and Green Bank telescopes. An "ARCC franchise" has recently begun at the University of Wisconsin at Milwaukee (Xavier Siemens, private communication).

In West Virginia, a collaboration between West Virginia University and the National Radio Astronomy Observatory has enabled a new initiative known as the Pulsar Search Collaboratory $^{5}$ (PSC). The PSC, which has been in existence since 2008, has involved high-school students from WV and surrounding states in pulsar search data from the GBT (Rosen et al. 2010). A unique aspect of this project is that the data are effectively "owned" by the students themselves and are not being analyzed by other groups. The students can therefore aim to make new discoveries and carry out the follow-up observations. Two discoveries so far have been made of transient sources, the first of which attracted significant interest from the White House with an invite to President Obama's star party ${ }^{6}$ for discoverer Lucas Bolyard.

\section{TRANSIENT SOURCES}

Isolated dispersed radio pulses from neutron stars have been one of the biggest new discoveries over the last decade or so. Following early work by Nice (1999) and Cordes \& McLaughlin (2001), it is now commonplace in pulsar searches to include a singlepulse detection module of the type described above. At Parkes, the discovery of the

\footnotetext{
${ }^{4}$ http://arcc.phys.utb.edu

${ }^{5} \mathrm{http}: / /$ pulsarsearchcollaboratory.com

${ }^{6}$ http://www.nrao.edu/pr/other/obama
} 


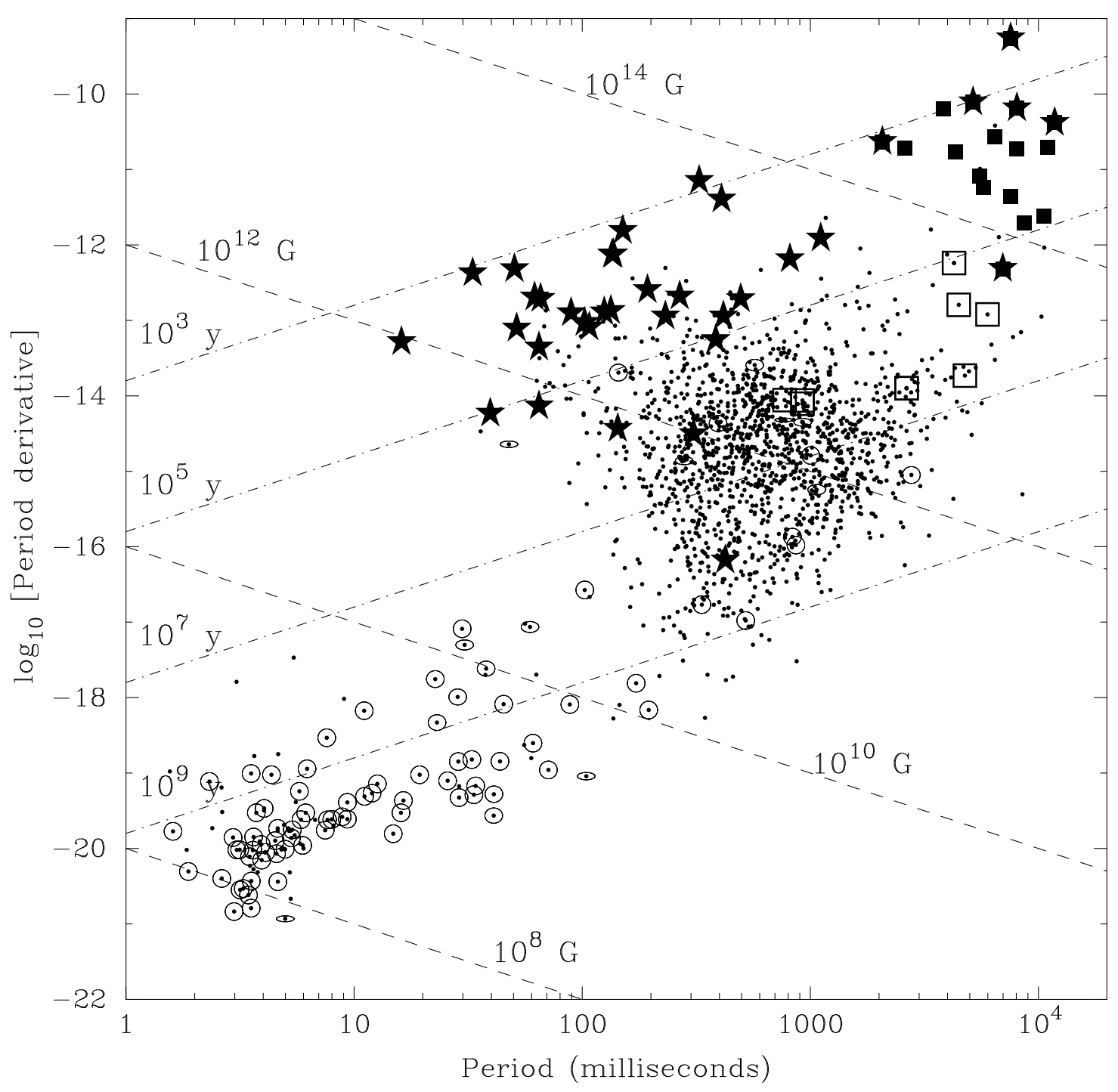

FIGURE 3. $\quad P-\dot{P}$ diagram showing normal pulsars as dots, binary pulsars as circles/ellipses, magnetars as filled squares, RRATs as open squares and supernova remnant associations as filled stars. Lines of constant characteristic age $\tau \propto P / \dot{P}$, magnetic field strength $B \propto \sqrt{P \dot{P}}$ are also shown.

rotating radio transients (RRATs) by McLaughlin et al. (2006) has opened up a new window on the neutron star population. Of the 30 RRATs currently known about a dozen now have measured spin-down rates and their location on the $P-\dot{P}$ diagram shown in Fig. 4. While there are many open questions as to how the RRATs fit into the pulsar population as a whole (see the contributions by Miller and Kaspi in these proceedings), one thing that is clear is that the RRATs manifest themselves in a number of ways. Some are perhaps just ordinary pulsars in which we see the tail end of the single-pulse amplitude distribution (e.g. Weltevrede et al. 2006), while some are clearly high $B$ and high $\dot{E}$ objects which show high-energy emission and appear to more closely resemble magnetars than pulsars. In my opinion, RRATs most likely represent 
a combination of neutron star source populations rather than a single class. While they may be observationally numerous as a whole, their birth rates need not add significantly to the total neutron star formation rate if they have evolved from other source classes which are already accounted for in birth-rate estimates. For further discussion and views, see Keane \& Kramer (2008).

\section{LESSONS LEARNED}

As these surveys continue to produce exciting individual objects and fill in our picture of the neutron star population, there are lessons to be learned which will be useful to remember as we move into the next era of searches with high-sensitivity facilities.

- Always expect the unexpected. The discovery of the RRATs is an excellent example of how blind surveys continue to surprise us. In general, casting as wide a net as possible when going on pulsar fishing expeditions is clearly a good thing.

- Always archive your data! The numerous re-analyses of the Parkes Multibeam surveys using different software, new techniques and different sets of eyes have really maximized the initial investment of telescope time for these surveys. Although pulsar search data are typically voluminous, it is extremely beneficial to invest in long-term archives which procure the data for future processing.

- Involve groups outside of your field. Our experience with high-school students has noticeably changed perceptions about science within the groups we have been working with and taps into a potentially vast resource of eyes that are willing to look for pulsars in new and exciting ways.

- Experiment with machine-based candidate ranking schemes. A number of groups are now actively applying the idea of neural network based approaches to deal with the vast amount of output that is now coming from pulsar searches (e.g. Eatough et al. 2010). As data rates, sensitivity and sky coverage increase over the coming years, this is set to become an even more important issue.

- Survey the sky multiple times with a given system. As the roles of pulsar intermittency and scintillation are becoming increasingly recognized as limiting factors in pulsar search sensitivity, multiple passes over each point in the sky can help mitigate these impacts. Surveys carried out with comparable sensitivity in different epochs (e.g. spaced by a decade) have also shown that pulsar detectability due to geodetic precession is an important factor (e.g. Burgay et al. 2006).

\section{BOLD PREDICTIONS FOR THE FUTURE}

In conclusion, I would say that blind searches for pulsars have an extremely promising future. Based on the current rate of progress of existing surveys, it is reasonable to aim for a doubling of the current population by 2015 (i.e. a sample of around 4000 pulsars). If surveys with more sensitive instruments such as FAST can be realized by 2015, I predict that as many as 10,000 pulsars could be found by 2020. Among these objects will surely be the long-awaited pulsar black-hole binary. As I tell the high-school students each 
year, the pulsar-black hole binary system could be discovered tomorrow by one of them! Another very tantalizing discovery would be pulsars orbiting Sgr A*. Despite multiple searches to date (Johnston et al. 2006; Deneva et al. 2009; Marquardt et al. 2010; Bates et al. 2010), no radio pulsar is currently known within 15 arcmin of the Galactic center. One of the great challenges is now to make the necessary sensitivity improvements to detect a faint radio pulsar in this region.

\section{ACKNOWLEDGMENTS}

I thank the organizers for putting together a fantastic programme of talks in a wonderful setting. My attendance at this meeting was enabled by a Research Challenge Grant from the West Virginia EPSCoR program. The ATNF pulsar catalogue (Manchester et al. 2005) was used to make Fig. 3, along with the McGill SGR/AXP Online Catalog ${ }^{7}$.

\section{REFERENCES}

1. S. D. Bates et al. 2010, MNRAS, in press.

2. M. Burgay et al. 2006, MNRAS, 368, 283.

3. M. Burgay et al. 2011, in preparation.

4. J. M. Cordes et al. 2006, ApJ, 637, 446.

5. J. M. Cordes, M. A. McLaughlin, 2003, ApJ, 596, 1142.

6. J. S. Deneva, J. M. Cordes, T. J. W. Lazio, 2009, ApJ, 702, L177.

7. R. P. Eatough et al. 2010, MNRAS, 407, 2443.

8. R. T. Edwards, M. Bailes, W. van Straten, W. \& M. C. Britton, 2001, MNRAS, 326, 358.

9. G. H. Janssen et al. 2009, A\&A, 498, 223.

10. G. Hobbs et al. 2009, PASA, 26, 468.

11. B. A. Jacoby, M. Bailes, S. M. Ord, R. T. Edwards, \& S. R. Kulkarni, 2009, ApJ, 699, 2009.

12. S. Johnston et al. 2006, MNRAS, 373, L6.

13. B. C. Joshi et al. 2009, MNRAS, 398, 943.

14. E. F. Keane \& M. Kramer 2008, MNRAS, 391, 2009.

15. M. J. Keith et al. 2010, MNRAS, 1356.

16. B. Knipsel et al., 2010, Science, 329, 1305.

17. L. Levin et al. 2010, ApJ, 721, L33.

18. F. Camilo et al. 2011, in preparation.

19. D. R. Lorimer, M. Kramer, 2005, "Handbook of Pulsar Astronomy”, Cambridge University Press.

20. R. N. Manchester et al. 2001, MNRAS, 328, 17.

21. R. N. Manchester et al. 2005, AJ, 129, 1993.

22. R. N. Manchester, G. Fan, A. G. Lyne, V. M. Kaspi \& F. Crawford, 2006, ApJ, 649, 235.

23. J. P. Marquardt et al. 2010, ApJ, 715, 939.

24. A. F. Miller, F. A. Jenet, A. Zermeno, K. Stovall, 2009, BAAS, 41, 264.

25. D. J. Nice, 1999, ApJ, 513, 927

26. S. M. Ransom et al. 2015, A\&A, in preparation.

27. R. Rosen et al. 2010, AER, 9, 010106-1.

28. L. Staveley-Smith et al. 1996, PASA, 13, 243.

29. P. Weltevrede et al. 2006, A\&A, 459, 597.

${ }^{7}$ http://www.physics.mcgill.ca/ pulsar/magnetar/main.html 